\title{
Co-Delivery of Prednisolone and Curcumin in Human Serum Albumin Nanoparticles for Effective Treatment of Rheumatoid Arthritis
}

This article was published in the following Dove Press journal: International Journal of Nanomedicine

\author{
Feili Yan $^{1}, *$ \\ Hui Li ${ }^{1} *$ \\ Zhirong Zhong ${ }^{1, *}$ \\ Meiling Zhou ${ }^{2}$ \\ Yan Lin' \\ Can Tang' \\ Chunhong $\mathrm{Li}^{\prime}$ \\ 'Department of Pharmaceutical Sciences, \\ School of Pharmacy, Southwest Medical \\ University, Luzhou 646000, Sichuan, \\ People's Republic of China; ${ }^{2}$ Department \\ of Pharmacy, The Affiliated Hospital of \\ Southwest Medical University, Luzhou, \\ Sichuan 646000, People's Republic of \\ China
}

*These authors contributed equally to this work
Correspondence: Can Tang; Chunhong Li Department of Pharmaceutical Sciences, School of Pharmacy, Southwest Medical University, 3-319 Zhongshan Road, Luzhou, Sichuan 646000, People's

Republic of China

Tel +86 I8982455783; +86 I3679696586

Fax +8608306302050

Email2291691527@qq.com;

lispringhong@126.com
Background: Prednisolone (PD) is extremely effective for treating rheumatoid arthritis (RA). However, it distributes nonspecifically throughout the body and its use is associated with serious side effects, which promoted us to compound it into a phytomedicine for greater efficacy and safety.

Methods: We combined PD with curcumin (CU), an effective monomer from traditional Chinese medicine, and human serum albumin (HSA) in a nanoparticulate system (N-PD/CU) to compensate for the poor bioavailability of PD and CU. N-PD/CU was prepared by highpressure homogenization, and its characteristics were evaluated in vitro. Next, we investigated its toxicity and mechanism of anti-inflammatory to macrophages. Finally, its pharmacokinetics, biodistribution and therapeutic efficacy were assessed in rats with adjuvantinduced arthritis (AIA)

Results: N-PD/CU showed a narrow size distribution around $150.4 \pm 2.4 \mathrm{~nm}$, a polydispersity index of $0.22 \pm 0.02$ and drug loading efficiency (DLE) of $88.75 \pm 1.82 \%$ for PD and $85.79 \pm 1.43 \%$ for CU. N-PD/CU showed sustained release of both drugs in vitro. $\mathrm{N}-\mathrm{PD} / \mathrm{CU}$ had no toxicity to macrophages in vitro on concentrations between 0.1 and $1.2 \mu \mathrm{mol} / \mathrm{mL}$. In activated macrophages, N-PD decreased levels of pro-inflammatory cytokines, while N-CU increased levels of anti-inflammatory IL-10, and N-PD/CU exhibited best therapeutic effect in vitro, suggesting co-delivery of PD and CU may synergistically control the course of RA. In AIA rats, N-PD/CU accumulated in inflamed joints through the effect of extravasation through leaky vasculature and subsequent inflammatory cell-mediated sequestration (ELVIS effect) in inflammatory lesion and showed higher therapeutic efficacy than single-loaded nanoparticles, either free drug on its own, or a simple mixture of the two drugs. Conclusion: This codelivery system based on HSA is a promising platform for combination chemotherapy in RA.

Keywords: co-delivery, prednisolone, curcumin, human serum albumin, rheumatoid arthritis

\section{Introduction}

Rheumatoid arthritis (RA) is a chronic systemic autoimmune disease of unknown etiology. It is characterized by symmetric, invasive inflammation in multiple joints of the hands and feet, causing pain and restricting movement. ${ }^{1}$ As the disease progresses, the risk of bone damage and cartilage destruction increases, leading to substantial disability. ${ }^{2,3}$

Pro-inflammatory cytokines such as TNF- $\alpha$, IL-1 $\beta$ and IL-6 drive progression of RA. ${ }^{4,5}$ IL-10, on the contrary, is an anti-inflammatory cytokine that can mitigate inflammation and immune responses in the disease. ${ }^{6}$ An effective therapy against 
the disease is glucocorticoids (GCs) such as prednisolone (PD), which can inhibit secretion of pro-inflammatory cytokines. ${ }^{7,8} \mathrm{PD}$, like most GCs, shows poor bioavailability because of its nonspecific distribution throughout the body, which increases the risk of adverse effects. ${ }^{9,10}$

This has motivated interest in using botanicals as adjunct therapy to prevent the development of arthritis and other chronic diseases. For example, the hydrophobic polyphenol curcumin (CU), from the rhizome of the herb Curcuma longa $L$., shows remarkable anti-inflammatory properties, and has shown efficacy against various diseases, including inflammatory disorders. ${ }^{11-13} \mathrm{CU}$ can attenuate RA by promoting the production of IL-10, which itself further reduces production of pro-inflammatory cytokines. ${ }^{14}$ However, like $\mathrm{PD}, \mathrm{CU}$ is hydrophobic, which limits their clinical application and their inability to target inflammatory lesion, cause adverse effects after administration. ${ }^{15-17}$ It may be that combining PD and CU can improve overall efficacy against RA, but this likely requires co-delivering them using a targeted system that can ensure that they act specifically in inflamed tissues, thereby reducing risk of adverse effects. ${ }^{18}$

An excellent nanocarrier candidate for these drugs is human serum albumin (HSA), which is nontoxic and biocompatible, ${ }^{18,19}$ and has high loading capacity for various drugs, allowing delivery of higher doses while keeping adverse events tolerable. ${ }^{20,21}$ In the present study, we examined whether we could deliver PD and CU effectively using HSA nanoparticles to inflamed joints in rats with adjuvant-induced arthritis (AIA). Our goal was to target the drugs to inflamed joints via the recently described phenomenon of extravasation through leaky vasculature and subsequent inflammatory cell-mediated sequestration (ELVIS effect). ${ }^{22-25}$ The ELVIS effect is, analogous to the classical enhanced permeability and retention (EPR) effect in solid tumors. ${ }^{26}$

We show here that HSA-based nanoparticles loaded with both PD and CU can be prepared by high-pressure homogenization and that in AIA rats, they accumulate specifically in inflamed joints and show greater antiinflammatory efficacy than the free drugs together or on their own.

\section{Materials and Methods}

\section{Materials}

Prednisolone (purity $\geq 99 \%$ ) was supplied by Chengdu Aike Pharmacetical (Chengdu, China); curcumin (purity $\geq 99 \%$ ),
Chengdu Mansite Biotechnology (Chengdu, China); oleic acid (OA) and purified yolk lecithin (lipoid E80), Lipoid Co., Ltd (Ludwigshafen, Germany); HSA and soybean oil, Beijing Biotopped Science \& Technology (Beijing, China); Incomplete Freund's adjuvant $(10 \mathrm{mg} / \mathrm{mL})$, Chondrex (Washington DC, USA). 3-(4,5-dimethylthiazol-2-yl)2,5-diphenyltetrazolium bromide (MTT) and Dioctadecyl tetramethyl Indodicarbocyanine (DID) were supplied by Solarbio Science \&Technology Co, Ltd (Beijing, China). Enzyme-linked immunosorbent assay (ELISA) kits to assay levels of TNF- $\alpha$, IL-1 $\beta$, IL- 6 and IL-10 in cell supernatant were purchased from Thermo Fisher (Vienna, Austria). ELISA kits to assay levels of TNF- $\alpha$, IL-1 $\beta$, IL-6 and IL-10 in serum of rats were obtained from Bioscience (San Diego, CA, USA). All other chemical reagents and solvents were of analytic grade or above.

\section{Cells and Animals}

RAW264.7 cells (Chinese Academy of Sciences, Shanghai, China) were cultured in RPMI 1640 medium (Hyclone Laboratories, Omaha, NE, USA) and supplemented with $10 \%(\mathrm{v} / \mathrm{v})$ fetal bovine serum (Hyclone) and $1 \%(\mathrm{v} / \mathrm{v})$ penicillin/streptomycin (Solarbio Science \& Technology, Beijing, China). Cells were incubated in a $5 \%$ $\mathrm{CO}_{2}$ environment at $37^{\circ} \mathrm{C}$.

Male Sprague-Dawley rats $(200 \pm 20 \mathrm{~g})$ were supplied by the Laboratory Animal Center of Southwest Medical University (Luzhou, China). All experimental rats were housed in common animal rooms and fed with standard feed, with indoor temperature $25 \pm 2{ }^{\circ} \mathrm{C}$ and relative wet degree $50 \pm 10 \%$. Animal experiments were approved by the Animal Ethics Committee of Southwest Medical University (Permit No. 20160126).During the whole animal experiments, laboratory animal guideline for ethical review of animal welfare for the animal was followed by according to National Standard of China.

\section{Preparation of Drug-Loaded Nanoparticles}

The lipophilicity of PD and CU was enhanced by forming PD-OA and CU-OA as described. ${ }^{27}$ Briefly, PD $(12 \mathrm{mg})$ and $\mathrm{CU}(6 \mathrm{mg})$ were dissolved in acetone $(6 \mathrm{~mL})$, to which was added $75 \mu \mathrm{L}$ OA $(10 \%$, w/v). The system was mixed well and stired up for one hour at room temperature, then acetone was removed by vacuum rotary evaporation. Prepared PD-OA and CU-OA, E80 (90 mg), and cholesterol (90 mg) were dissolved in chloroform and mixed together, then HSA 
solution at a concentration of $1.3 \%(\mathrm{w} / \mathrm{v})$ was added. Then the mixture was subjected to high-speed shearing for $1 \mathrm{~min}$ (Bio-Homogenizer, Biospec Products, USA), followed by homogenization into a emulsion at $18,000 \mathrm{psi}$ for 10 cycles (AH 100D; ATS Engineering, Canada). Finally, the organic solvent was removed by vacuum rotary evaporation. The resulting nanoparticles, loaded with both drugs, were referred to as $\mathrm{N}-\mathrm{PD} / \mathrm{CU}$. The same method was used to prepare blank nanoparticles without either drug, as well as nanoparticles with each drug on its own (N-PD, N-CU).

\section{Characteristics of Drug-Loaded Nanoparticles}

The size and zeta potential of drug-loaded HSA nanoparticles were measured at room temperature using a Malvern Zetasizer Nano ZS90 (Malvern Instruments, Malvern, UK), using equal volumes of nanoparticles suspended in ultrapure water. Samples of nanoparticles were also analyzed by transmission electron microscopy (H-600, Hitachi, Japan). Samples were diluted in ultrapure water and stained with phosphotungstic acid for approximately $30 \mathrm{~s}$. Encapsulation efficiency (EE) and drug loading efficiency (DLE) were measured by HPLC.

\section{Drug Release from HSA Nanoparticles in vitro}

Dialysis was used to analyze the release of PD and CU from N-PD/CU. Dialysis bags (molecular weight cut-off, $10 \mathrm{kDa}$ ) were filled with $1.5 \mathrm{~mL}$ of N-PD/CU or the equivalent amounts of either free drug, and the bags were immersed in $10 \mathrm{~mL}$ of phosphate-buffered saline (PBS) containing $0.2 \%(\mathrm{w} / \mathrm{v})$ Tween 80 with stirring at $100 \mathrm{rpm}$ at $37^{\circ} \mathrm{C} .{ }^{19}$ At fixed times, a sample of release medium $(300 \mu \mathrm{L})$ was extracted and the same volume of fresh medium was added back to keep the final volume constant. Concentrations of each drug in the release medium were determined by HPLC (Agilent 1260, USA).

\section{Cytotoxicity of Nanoparticles Toward RAW264.7 Cells in vitro}

RAW264.7 cells were seeded into 96-well plates at a density of $1 \times 10^{4}$ per well and incubated in $5 \% \mathrm{CO}_{2}$ at $37^{\circ} \mathrm{C}$. After $24 \mathrm{~h}$, the original medium was replaced with $200 \mu \mathrm{L}$ of solutions containing various concentrations of blank nanoparticles, N-PD/CU, single-loaded nanoparticles, each free drug on its own, or a simple mixture of both free drugs. Cells were incubated for another $48 \mathrm{~h}$, then the numbers of living cells were determined using the MTT assay. ${ }^{19}$ Relative cell viability was calculated based on the absorption of treated wells relative to that of control wells incubated with drug-free medium.

\section{Pharmacodynamics in vitro}

RAW264.7 cells in logarithmic growth phase were digested into a single-cell suspension, inoculated into 6 -well plates $\left(5 \times 10^{5}\right.$ cells per well), then cultured for 24 h. Cells were incubated in a $5 \% \mathrm{CO}_{2}$ atmosphere at $37^{\circ} \mathrm{C}$. Then LPS was added to a final concentration of $100 \mathrm{ng} / \mathrm{mL}$ and cultured for $24 \mathrm{~h}$ to activate the cells. The medium was replaced with $2 \mathrm{~mL}$ of fresh medium containing free $\mathrm{PD}$, free $\mathrm{CU}, \mathrm{N}-\mathrm{PD}, \mathrm{N}-\mathrm{CU}, \mathrm{N}-\mathrm{PD} / \mathrm{CU}$, or the simple combination of $\mathrm{PD}$ and $\mathrm{CU}$ (total drug concentration in all cases was $200 \mu \mathrm{g} / \mathrm{mL}$ ). After incubation for $48 \mathrm{~h}$, the medium was collected, centrifuged at $2000 \mathrm{rpm}$ for $4 \mathrm{~min}$, and the supernatant was assayed for the inflammatory cytokines TNF- $\alpha$, IL-6, IL-1 $\beta$ and IL-10, using ELISA kits according to the manufacturer's protocols. ${ }^{28}$

\section{Pharmacokinetics and Biodistribution of Nanoparticles}

Fifty male Sprague-Dawley rats $(200 \pm 20 \mathrm{~g})$ were used to established a rat model of AIA. Heat-killed mycobacteria were suspended in incomplete Freund's adjuvant $(10 \mathrm{mg} /$ $\mathrm{mL}$ ), then each rat was injected subcutaneously with $100 \mu \mathrm{L}$ at the base of the tail. Every day, we evaluated the articular index (AI) score for the paws of each rat as described. ${ }^{7}$ After 14 days, we chose the AIA rats with an AI score $>4$ points to study the in vivo pharmacokinetics and distribution. The selected rats were fasted and randomly assigned to 10 groups $(n=5)$. Five groups received a single injection of N-PD/CU containing $5.32 \mathrm{mg} / \mathrm{kg}$ PD and $2.55 \mathrm{mg} / \mathrm{kg} \mathrm{CU}$, while the other five groups received a single injection of a simple mixture of PD and $\mathrm{CU}$ at the same concentrations as in the nanoparticles. Blood samples were collected at 0.25, 0.5, 1, 2 and $4 \mathrm{~h}$, and concentrations of each drug in serum were determined by HPLC. Meanwhile, various tissues were collected (heart, liver, spleen, lung, kidney and joints), homogenized in a volume of $0.9 \% \mathrm{NaCl}$ equal to twice the tissue weight, and assayed for each drug by HPLC.

\section{Distribution of N-PD/CU in Arthritic Rats}

Nine AIA rats were depilated over the entire body using cream, and then given a tail vein injection of saline, free DID or DID/N-PD/CU (5 $\mu$ g DID per rat). At 4, 12, and 24 
$\mathrm{h}$, animals were anesthetized with $10 \%$ chloral hydrate and imaged using a real-time fluorescence imaging system ( $\mathrm{Fx}$ Pro/FX, Bruker, USA). ${ }^{29}$

\section{Therapeutic Efficacy of Nanoparticles in}

\section{Arthritic Rats}

On days 14, 16, 18, 20 and 22 after arthritis induction, rats in each group $(\mathrm{n}=3)$ were injected intravenously with N-PD/ $\mathrm{CU}, \mathrm{N}-\mathrm{PD}, \mathrm{N}-\mathrm{CU}$, either free drug on its own, a simple mixture of the two free drugs, or saline. The PD dose was $5.32 \mathrm{mg} / \mathrm{kg}$, and the CU dose was $2.55 \mathrm{mg} / \mathrm{kg}$. Three healthy rats not receiving any treatment served as a naive control.

On every other day starting on day 14 after arthritis induction, swelling was assessed in each limb of all animals as described ${ }^{7}$ and we summed all scores for each animal to obtain an overall score.

On day 22 after arthritis induction, right ankle joints were dissected from rats in each of the groups. Hind paws were fixed in $4 \%$ paraformaldehyde for $72 \mathrm{~h}$, decalcified with $15 \%$ neutral EDTA solution for 60 days at room temperature, then embedded in paraffin. Thin sections were cut and stained with hematoxylin-eosin.

\section{Pro-Inflammatory Cytokine and Anti-Inflammatory Expression in Serum of Arthritic Rats After Nanoparticles}

\section{Treatment}

On day 22 after arthritis induction, blood was collected from arthritic rats in each of the groups. Serum levels of pro-inflammatory cytokines (TNF- $\alpha$, IL- $1 \beta$ and IL-10) and the anti-inflammatory cytokine IL-10 were measured using ELISA kits according to the manufacturer's instructions.

\section{Statistical Analysis}

Statistical analysis was performed using SPSS 19.0 (IBM, Chicago, IL, USA). Differences between two groups were assessed for significance using Student's $t$ test, and differences among more than two groups were assessed using ANOVA. Differences associated with $p<0.05$ were considered significant. All results were expressed as mean $\pm \mathrm{SD}$.

\section{Results}

\section{Characterization of Drug-Loaded}

\section{Nanoparticles}

Dynamic light scattering showed the average hydrodynamic diameter of N-PD/CU to be $150.4 \pm 2.4 \mathrm{~nm}$; N-PD, $136.90 \pm$
$5.0 \mathrm{~nm}$; and N-CU, $148.0 \pm 3.1 \mathrm{~nm}$ (Figure 1A-C). Blank nanoparticles were smallest (Figure 1D). The polydispersity index (PDI) was less than 0.3 for all nanoparticles (Figure 1A), indicating uniform size. Average zeta potential was $-21.6 \pm 1.9 \mathrm{mV}$ for $\mathrm{N}-\mathrm{PD} / \mathrm{CU},-18.6 \pm 1.9 \mathrm{mV}$ for $\mathrm{N}-\mathrm{PD},-25.2 \pm 2.6 \mathrm{mV}$ for $\mathrm{N}-\mathrm{CU}$, and $-26.5 \pm 2.2 \mathrm{mV}$ for blank nanoparticles (Figure 2A). Transmission electron microscopy showed that all types of nanoparticles were monodisperse and uniformly spherical (Figure 1E), with sizes of 100-200 nm. Drug encapsulation efficiency of $\mathrm{N}-\mathrm{PD} / \mathrm{CU}$ was $88.75 \pm 1.82 \%(\mathrm{w} / \mathrm{w})$ for PD and $85.79 \pm$ $1.43 \%(\mathrm{w} / \mathrm{w})$ for $\mathrm{CU}$, for a total drug loading efficiency of $2.36 \pm 0.04 \%(\mathrm{w} / \mathrm{w})$ (Figure 2A). Encapsulation and drug loading efficiencies of N-PD were, respectively, $91.61 \pm$ $1.82 \%$ and $2.30 \pm 0.03 \%$. N-CU showed slightly lower encapsulation and drug loading efficiencies.

Average zeta potential was $-21.6 \pm 1.9 \mathrm{mV}$ for N-PD/ $\mathrm{CU},-18.6 \pm 1.9 \mathrm{mV}$ for nanoparticles containing only PD, $-25.2 \pm 2.6 \mathrm{mV}$ for nanoparticles containing only $\mathrm{CU}$, and $-26.5 \pm 2.2 \mathrm{mV}$ for blank nanoparticles (Figure 2A). Transmission electron microscopy showed that all types of nanoparticles were monodisperse and uniformly spherical (Figure 1E), with sizes of 100-200 nm. Drug encapsulation efficiency of N-PD/CU was $88.75 \pm 1.82 \%$ (w/w) for PD and $85.79 \pm 1.43 \%(\mathrm{w} / \mathrm{w})$ for $\mathrm{CU}$, for a total drug loading efficiency of $2.36 \pm 0.04 \%(\mathrm{w} / \mathrm{w})$ (Figure 2A). Encapsulation and drug loading efficiency of N-PD were respective $91.61 \pm 1.82 \%$ and $2.30 \pm 0.03 \%$, which were both a bit higher than N-CU.

\section{Drug Release from Nanoparticles in vitro} Compared to the free drug solution, $\mathrm{PD}$ and $\mathrm{CU}$ were released more slowly from N-PD/CU. By around $10 \mathrm{~h}$, nearly $100 \%$ of free $\mathrm{CU}$ was released from the corresponding free drug solution, compared to $24 \mathrm{~h}$ for PD. In contrast, by $72 \mathrm{~h}$, only $49.22 \pm 1.95 \%$ of PD had been released from N-PD/CU (Figure 2B), similar to $48.72 \pm 1.24 \%$ of $\mathrm{CU}$ (Figure 2C). These results indicate that N-PD/CU allows controlled drug release.

\section{Nanoparticles Cytotoxicity in vitro}

Cell viability remained about $94 \%$ after exposure to HSA concentrations between 0.1 and $1.2 \mu \mathrm{mol} / \mathrm{mL}$ (Figure 3). Cells exposed to N-PD/CU showed similar viability as those exposed to blank nanoparticles, suggesting that the HSA-based drug carrier is nontoxic. In fact, cells exposed to $\mathrm{N}-\mathrm{PD} / \mathrm{CU}$ showed higher viability than cells exposed to a simple mixture of both free drugs. 

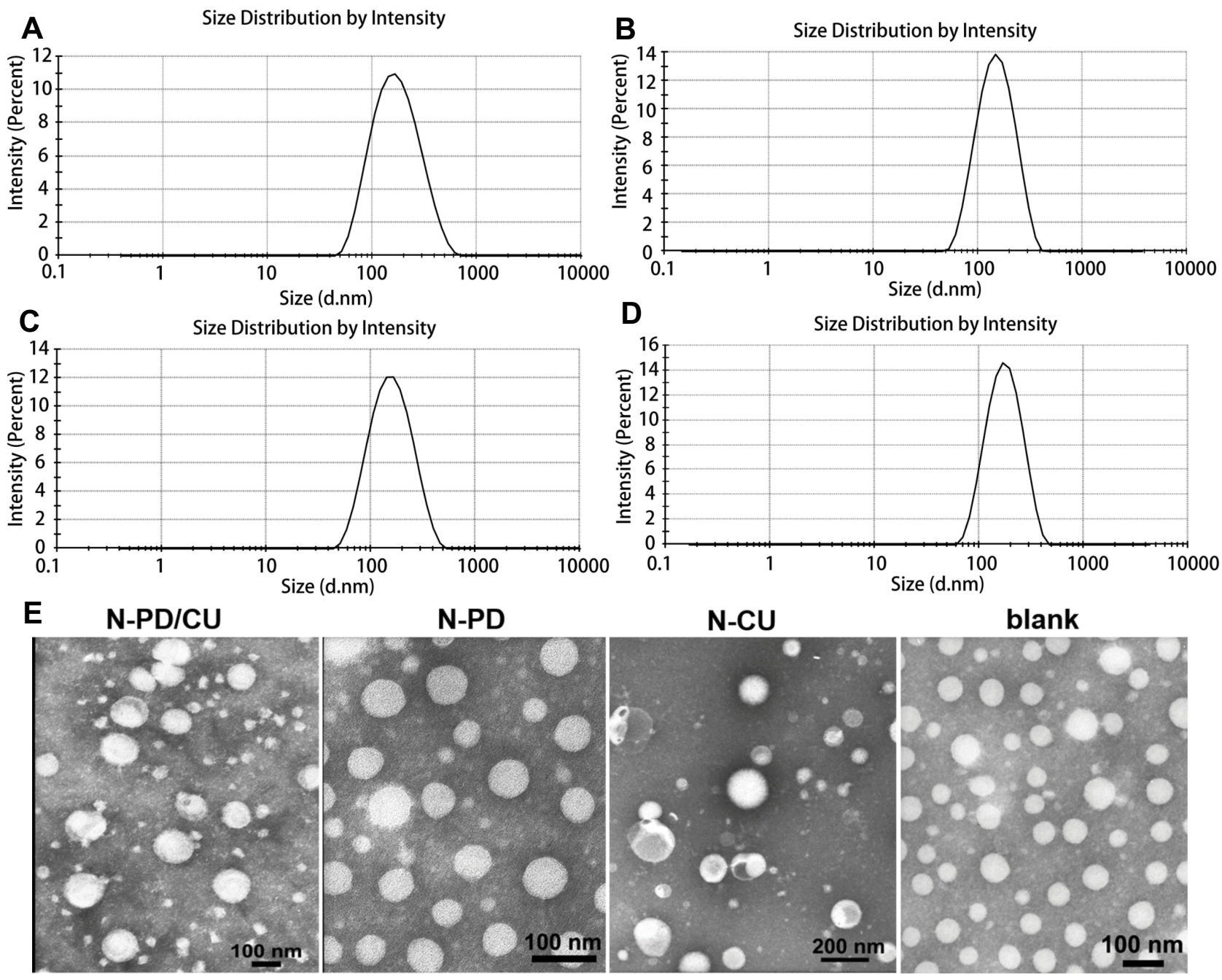

Figure I Size of N-PD/CU (A), N-PD (B), N-CU (C) and blank nanoparticles (D). (E) Morphologies of different human serum albumin nanoparticles as measured by TEM.

\section{Pharmacodynamics in vitro}

To observe the anti-inflammatory effects of N-PD/CU on activated macrophages, levels of inflammatory cytokines were measured after various treatments. The N-PD/CU group showed significantly lower levels of TNF- $\alpha$ and IL-6 in the supernatant after spinning down cultured cells than other groups (Figure 4A and $\mathrm{C},{ }^{*} p<0.05,{ }^{* *} p<0.001$ or $* * * p<0.0001)$, and it showed significantly lower levels of IL-1 $\beta$ than the N-CU group (Figure $4 \mathrm{~B},{ }^{*} p<0.05$ ). Conversely, the N-PD/CU group showed significantly higher levels of IL-10 than other groups except N-CU (Figure $4 \mathrm{D},{ }^{*} p<0.05$ or ${ }^{* *} p<0.001$ ). These results suggest that N-PD/CU can inhibit the production of proinflammatory cytokines and promote the release of antiinflammatory IL-10 to a greater extent than single-loaded nanoparticles, either free drug on its own, or a simple mixture of the two drugs.
To explore in greater detail how the drug cargoes, PD and $\mathrm{CU}$, work against $\mathrm{RA}$ in activated macrophages, we analyzed the effects of N-PD and N-CU on inflammatory cytokine levels. N-PD led to significantly lower levels of TNF- $\alpha$ and IL- $1 \beta$ than N-CU compared to PBS (Figure 4A and $\left.\mathrm{B},{ }^{\#} p<0.05,{ }^{\# \#} p<0.001,{ }^{\# \#} p<0.001\right)$, while N-CU led to significantly higher levels of IL-10 than N-PD compared to PBS (Figure 4D, ${ }^{\#} p<0.05$ ). These results suggest that PD primarily inhibits the production of pro-inflammatory cytokines TNF- $\alpha$ and IL- $1 \beta$, while CU effectively promotes the production of anti-inflammatory cytokine IL-10.

\section{Biodistribution and Pharmacokinetics of Nanoparticles}

Encapsulating PD and $\mathrm{CU}$ in nanoparticles significantly increased their concentrations in liver, spleen, and joints at $15 \mathrm{~min}, 1 \mathrm{~h}, 2 \mathrm{~h}$ and $4 \mathrm{~h}$ after injection into AIA rats 


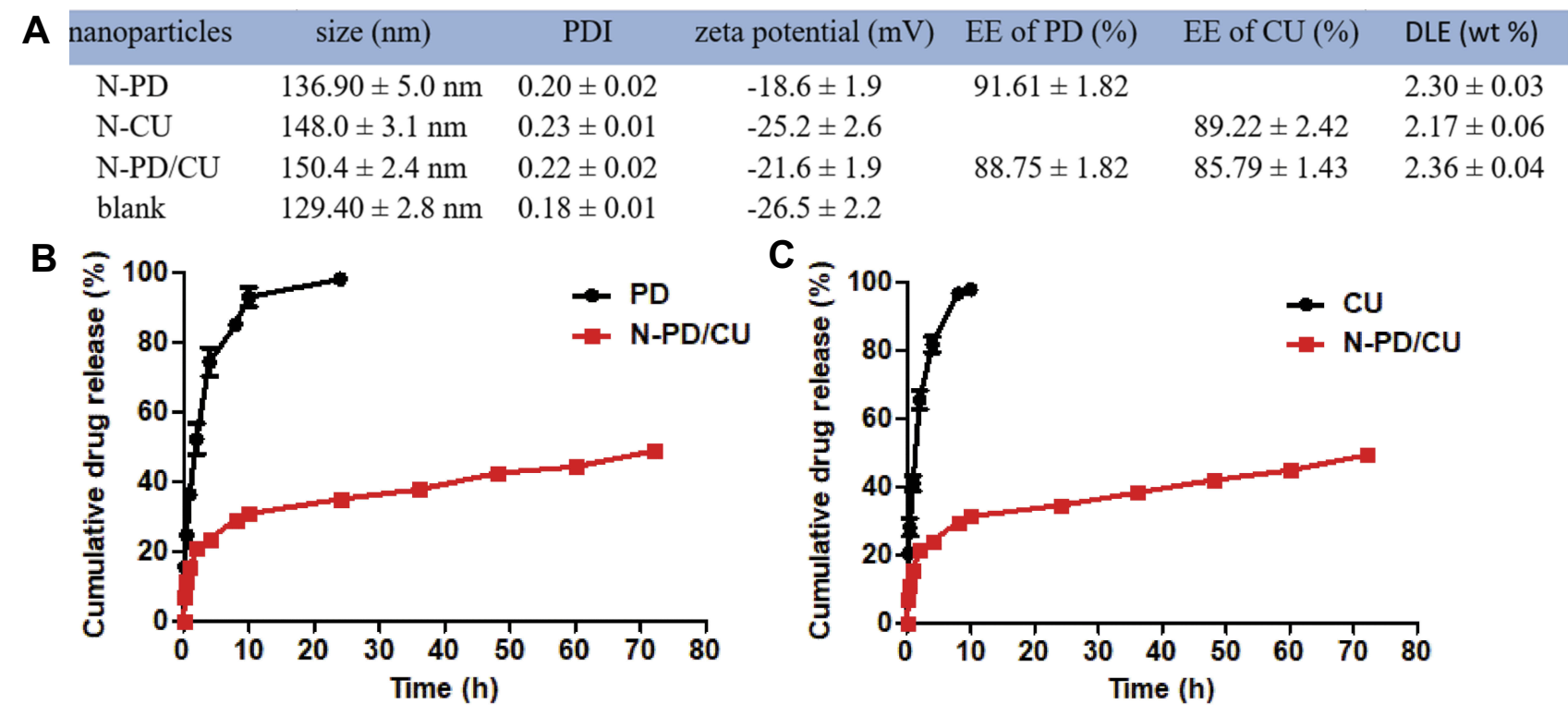

Figure 2 Characteristic of nanoparticles. (A) Size, PDI, zeta potential EE and DLE of nanoparticles, Data represent the mean \pm SD ( $\mathrm{n}=3$ ). (B) Cumulative release of PD from N-PD/CU and free PD solution. (C) Cumulative release of CU from N-PD/CU and free CU solution. Data represent the mean \pm SD ( $n=3$ ).

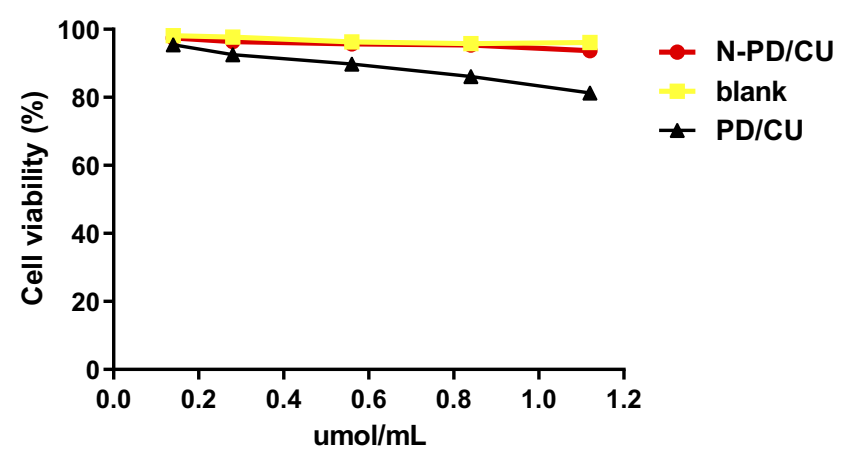

Figure 3 The effect of N-PD/CU, blank nanoparticle and PD/CU for RAW264.7 cells viability. Data represent mean $\pm S D(n=5)$.

(Figure 5A). In fact, the nanoparticles increased drug concentration in joints relative to the simple drug mixture by 2.1 -fold at $15 \min \left({ }^{*} p<0.05\right), 3.5$-fold at $1 \mathrm{~h}(* * p<0.001)$, 6.6-fold at $2 \mathrm{~h}(* * * p<0.0001)$, and 8.4 -fold at 4 $\mathrm{h}\left({ }^{* *} p<0.001\right) . \mathrm{AUC}_{0-4} \mathrm{~h}$ of $\mathrm{PD}$ in joints was significantly higher with N-PD/CU $(6.61 \pm 0.57 \mu \mathrm{g} / \mathrm{g} \cdot \mathrm{h})$ than with the simple mixture of both drugs $(1.92 \pm 0.10 \mu \mathrm{g} / \mathrm{g} \cdot \mathrm{h}, p<0.05)$. Furthermore, there was no significant difference in drug levels between the two treatment groups in heart or lung at any of the time points (Figure 5A, $p>0.05$ ). Levels of the drugs were similar between the two treatment groups at $0.25,1$ and $2 \mathrm{~h}$ (Figure 5A, $p>0.05$ ), but the level of N-PD/ $\mathrm{CU}$ was higher than the level of the simple mixture at $4 \mathrm{~h}$.

Biodistribution of N-PD/CU was significantly higher than $\mathrm{N}-\mathrm{CU}$ in heart, liver, spleen, and joints at $15 \mathrm{~min}, 1 \mathrm{~h}$, $2 \mathrm{~h}$ and $4 \mathrm{~h}$ (Figure 5B). AUC $0-4 \mathrm{~h}$ was significantly higher for N-PD/CU $(2.20 \pm 0.25 \mu \mathrm{g} / \mathrm{g} \cdot \mathrm{h})$ than for the drug mixture $\left(0.71 \pm 0.04 \mu \mathrm{g} / \mathrm{g} \cdot \mathrm{h},{ }^{*} p<0.05\right)$.

By analyzing drug concentration-time profiles, we found that encapsulating PD into N-PD/CU led to much higher PD concentrations in the plasma at early time points (Figure 6A). The area under the curve (AUC) and $\mathrm{C}_{\max }$ were, respectively, 2.62- and 2.31-fold higher for $\mathrm{N}-\mathrm{PD} / \mathrm{CU}$ than for the simple mixture of both free drugs. Similar results were obtained for CU (Figure 6B). Furthermore, $\mathrm{t}_{1 / 2}$ for N-PD/CU was 2.36 times higher than for N-PD and 1.25 times higher than for N-CU (Figure 6C).

Figure 7 compares the pharmacokinetics of N-PD/CU with the pharmacokinetics of the simple mixture of two drugs in the plasma of AIA rats. In the case of both drugs, AUC and $\mathrm{C}_{\max }$ were significantly higher for N-PD/CU than for the simple mixture. In animals treated with $\mathrm{N}-\mathrm{PD} / \mathrm{CU}, \mathrm{Re}_{\text {joint }}$ and $\mathrm{Ce}_{\text {joint }}$ were 3.44 and 2.13 for PD and 3.10 and 2.82 for CU. These results indicate that HSA-based nanoparticles lead to greater joint accumulation of both drugs.

\section{Distribution of N-PD/CU in Arthritic Rats}

Real-time fluorescence imaging showed that DID/N-PD/CU accumulated in joints more than free DID (Figure 8). At 4 $\mathrm{h}$ and $12 \mathrm{~h}, \mathrm{DID} / \mathrm{N}-\mathrm{PD} / \mathrm{CU}$ showed detectable fluorescence in 


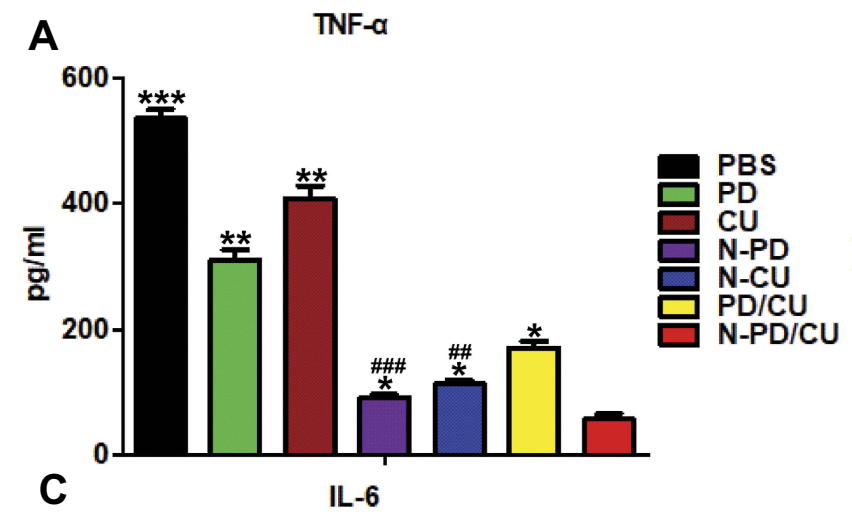

B IL-1
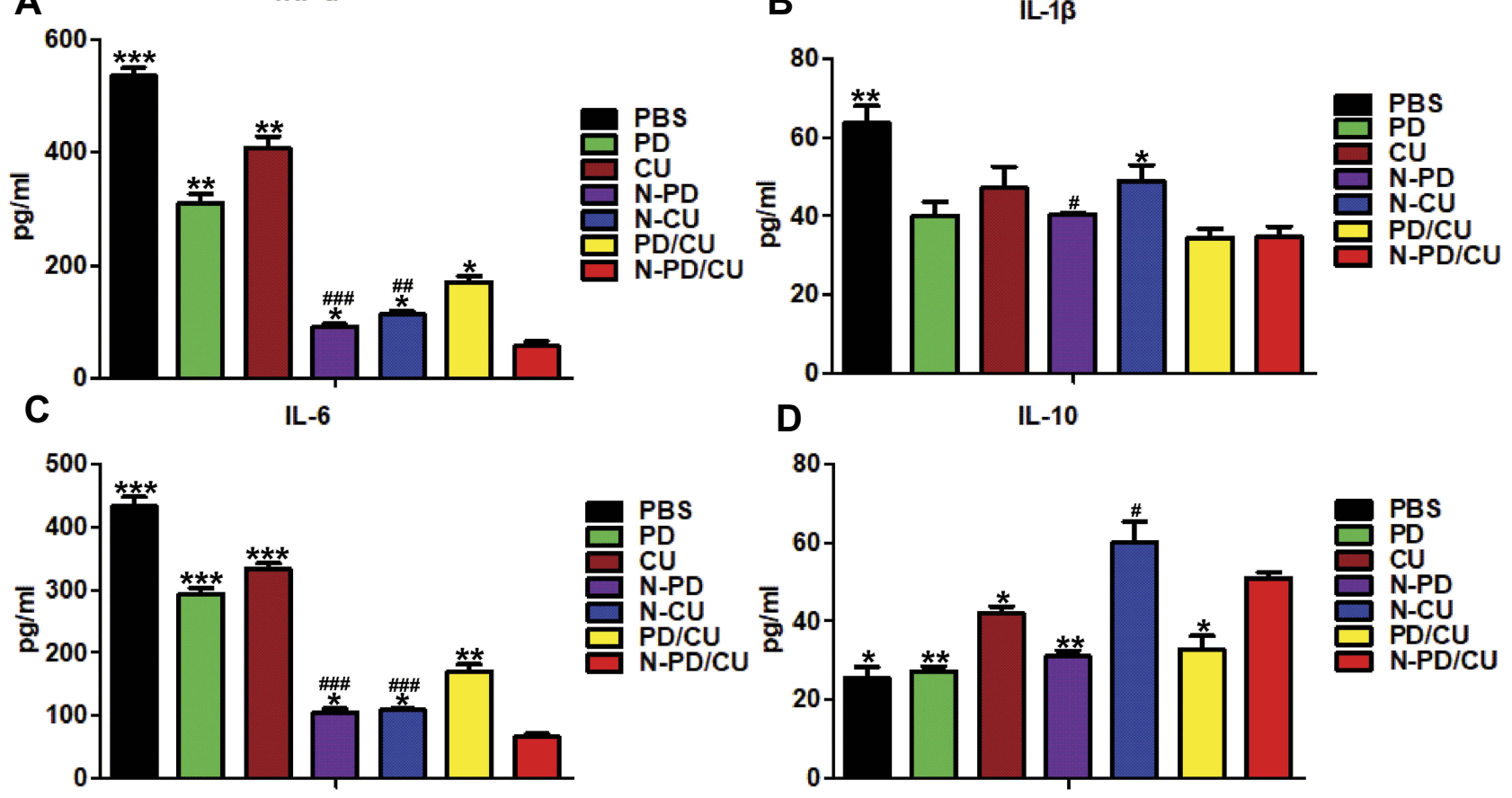

Figure 4 Expression of pro-inflammatory cytokines and anti-inflammatory cytokine in cell-free supernatant of activated macrophage treated with different preparations. Levels of (A) TNF- $\boldsymbol{\alpha},(\mathbf{B})$ IL-I $\boldsymbol{\beta},(\mathbf{C})$ IL-6 and (D) IL-I0 were assayed. Data shown are mean \pm SD $(\mathrm{n}=3)$. $*_{p}<0.05, *^{*} p<0.00$ I, $* * * p<0.000$ I vs cells treated with N-PD/CU, ${ }^{\#} p<0.05,{ }^{\#} p<0.001,{ }^{\#} p<0.0001$ vs cells treated with PBS.

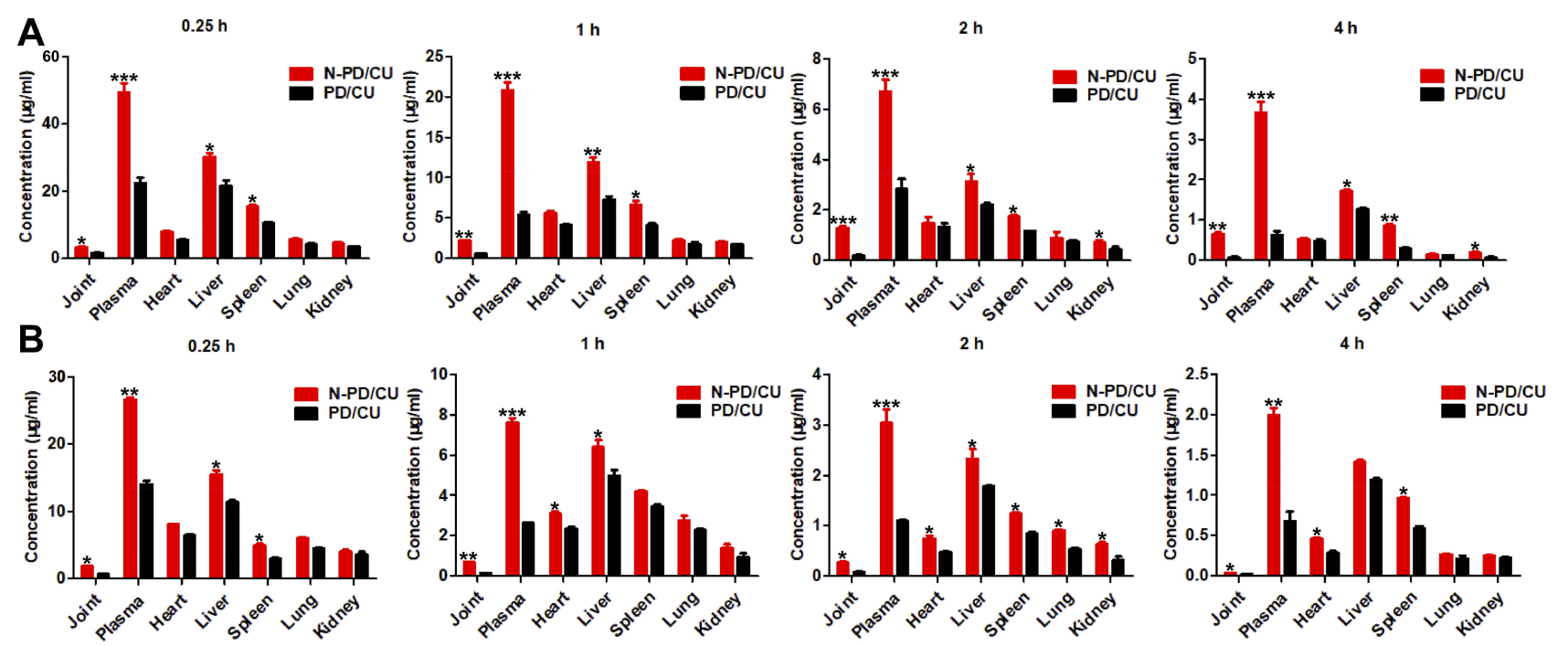

Figure 5 Biodistribution profiles of N-PD/CU and PD/CU. (A)The concentration of PD in different plasma, organs and joints at different time points in AIA rats treated by $\mathrm{N}-\mathrm{PD} / \mathrm{CU}$ and PD/CU. (B)The distribution of $\mathrm{CU}$ in different plasma, organs and joints at different time points in AIA rats treated with N-PD/CU and PD/CU. Data represent the mean $\pm S D(n=5)$. $* p<0.05$, $* * p<0.001$, *** $p<0.0001$ vs animals treated with PD/CU.

the hind limbs of AIA rats, whereas free DID did not. Even at $24 \mathrm{~h}$, most free DID fluorescence was in internal organs. These results suggest that N-PD/CU can targets inflammatory lesions, confirm with the results of pharmacokinetics that HSA-based nanoparticles lead to greater joint accumulation.

\section{Therapeutic Efficacy of Nanoparticles} in vivo

Arthritic rats were treated by tail vein injection with saline, free PD, free CU, N-PD/CU, N-PD or N-CU. Each group was monitored for changes in body weight, swelling 

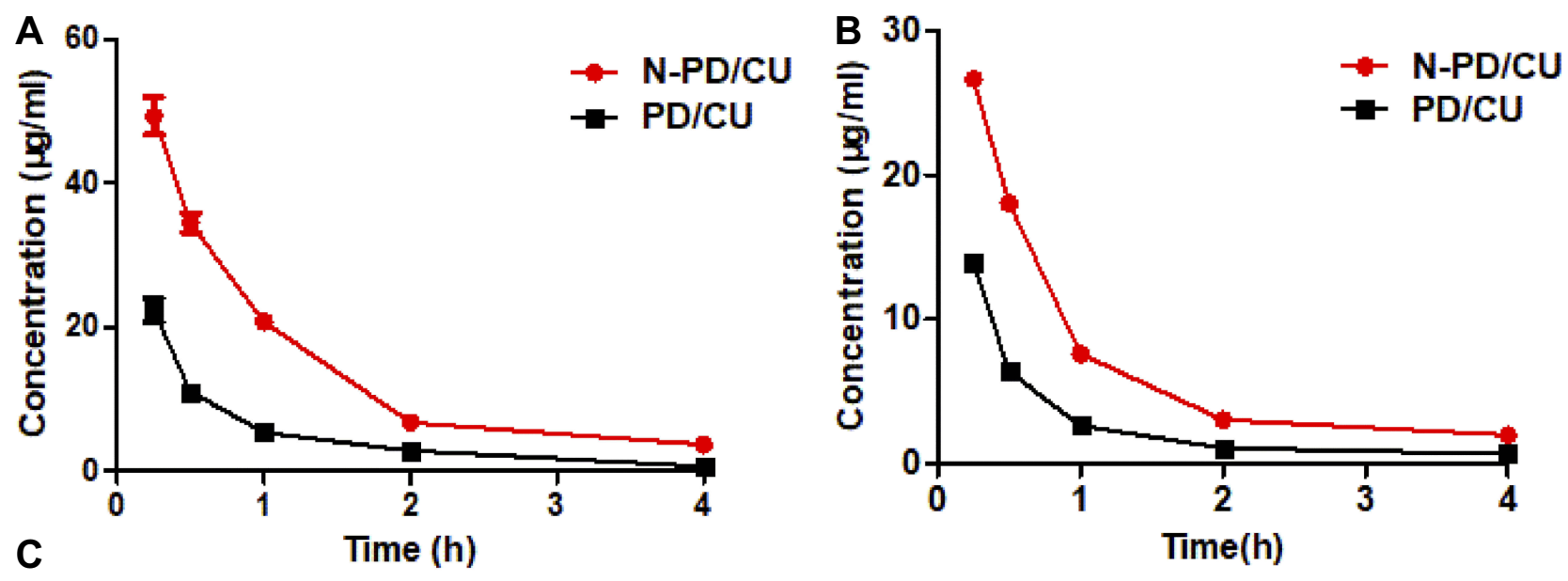

\begin{tabular}{lcccc}
\hline & \multicolumn{2}{c}{$\mathrm{N}-\mathrm{PD} / \mathrm{CU}$} & \multicolumn{2}{c}{$\mathrm{PD} / \mathrm{CU}$} \\
\cline { 2 - 5 } parameters & $\mathrm{PD}$ & $\mathrm{CU}$ & $\mathrm{PD}$ & $\mathrm{CU}$ \\
\hline $\mathrm{AUC}_{0-\mathrm{t}}(\mu \mathrm{g} / \mathrm{g} . \mathrm{h})$ & $63.29 \pm 5.13^{\mathrm{a}}$ & $30.28 \pm 1.14^{\mathrm{b}}$ & $24.17 \pm 3.13$ & $14.12 \pm 1.24$ \\
$\mathrm{Cmax}(\mu \mathrm{mol} / \mathrm{g})$ & $49.62 \pm 6.60^{\mathrm{a}}$ & $26.62 \pm 0.69^{\mathrm{b}}$ & $21.51 \pm 3.58$ & $14.01 \pm 1.34$ \\
$\mathrm{~T}_{1 / 2}(\mathrm{~h})$ & $1.20 \pm 0.27^{\mathrm{a}}$ & $0.67 \pm 0.04^{\mathrm{b}}$ & $0.73 \pm 0.18$ & $0.56 \pm 0.14$ \\
\hline
\end{tabular}

Abbreviations: $\mathrm{AUC}_{0-\mathrm{t}}$ : area under drug concentration-time curve, $\mathrm{C}_{\max }$ : maximum drug concentration.

$$
\begin{aligned}
& { }^{\mathrm{a}} p<0.05 \text { versus } \mathrm{PD} \text { of } \mathrm{PD} / \mathrm{CU} \text { group. } \\
& { }^{\mathrm{b}} p<0.05 \text { versus } \mathrm{CU} \text { of } \mathrm{PD} / \mathrm{CU} \text { group. }
\end{aligned}
$$

Figure 6 Plasma concentration-time curves of N-PD/CU and PD/CU. (A) Concentration-time curves of PD. (B) Concentration-time curves of CU. Data represent the mean \pm SD $(n=5)$. (C) Pharmacokinetic parameters of N-PD/CU and PD/CU $(n=5)$.

scores and histopathology. Histological sections of joints in the right hind limb were analyzed under the microscope, using healthy rats as a control group.

Body weight in rats injected with N-PD/CU increased continuously from day 14 (booster injection) to day 22; their body weight was similar to that of healthy, untreated rats (Figure 9A). Mean arthritis scores were lowest in animals treated with N-PD/CU (Figure 9B), which showed barely noticeable swelling of joints. In the joints of rats treated with saline, histological examination of right hind limbs revealed massive synovial cell lining, extensive pannus formation and severe bone destruction (Figure 9C); the histopathology score was highest $(>7.0)$ in this group. Animals treated with either free drug showed much thicker synovial cell lining and greater bone destruction than animals treated with the corresponding single-loaded nanoparticles. Animals treated with either free drug showed similar histopathology scores as those treated with saline. Animals treated with a simple mixture of the two drugs showed less synovial cell lining and less severe bone destruction, with a histopathology score of 4.0. Rats treated with N-PD/CU showed minor pannus formation and obvious bone protection, with a histopathology score of 2.0.

\section{Effects of Nanoparticles on Pro-Inflammatory and Anti-Inflammatory Cytokine Production in vivo}

The cytokines TNF- $\alpha$, IL- $1 \beta$, and IL- 6 drive progression of rheumatoid arthritis, while anti-inflammatory cytokines slow progression. Levels of all three pro-inflammatory cytokines in serum were significantly lower in animals treated with $\mathrm{N}-\mathrm{PD} / \mathrm{CU}$ than in animals in other groups $\left({ }^{*} p<0.05,{ }^{* *} p<0.001\right.$ or ${ }^{* * *} p<0.0001$, Figure $\left.10 \mathrm{~A}-\mathrm{C}\right)$. Conversely, IL-10 levels in serum were significantly higher in animals treated with $\mathrm{N}-\mathrm{PD} / \mathrm{CU}$ than in animals in other groups $\left(* * p<0.001\right.$ or ${ }^{* * *} p<0.0001$, Figure 10D). 


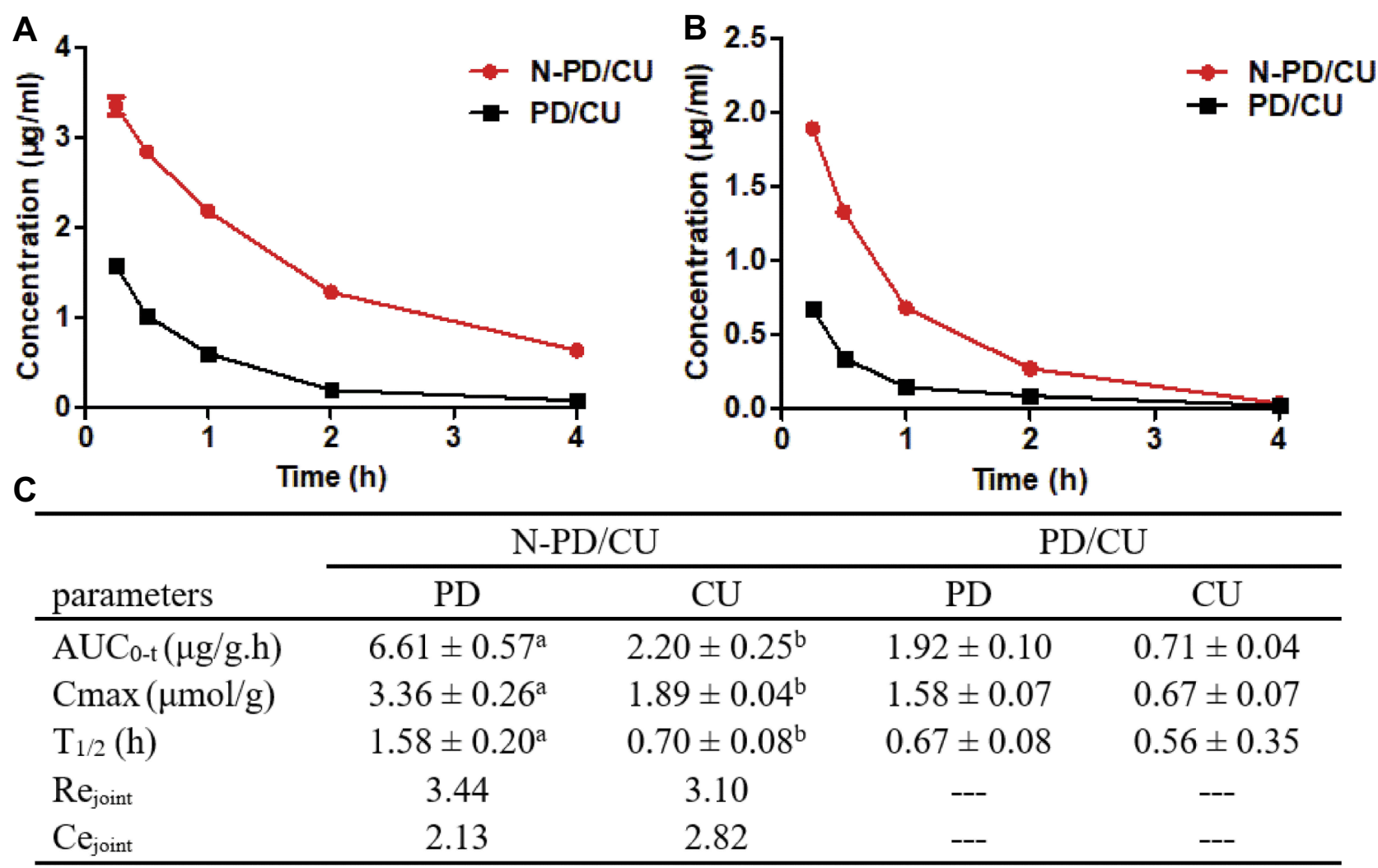

Abbreviations: $\mathrm{AUC}_{0-\mathrm{t}}$ : area under drug concentration-time curve, $\mathrm{C}_{\max }$ : maximum drug concentration, $\mathrm{Re}_{\text {joint }}$ : relative uptake efficiency, $\mathrm{Ce}_{\text {joint }}$ : concentration efficiency.

${ }^{\text {a }} p<0.05$ versus $\mathrm{PD}$ of $\mathrm{PD} / \mathrm{CU}$ group.

${ }^{\mathrm{b}} p<0.05$ versus $\mathrm{CU}$ of $\mathrm{PD} / \mathrm{CU}$ group.

Figure 7 Joints concentration-time curves of N-PD/CU and PD/CU. (A) Concentration-time curves of PD. (B) Concentration-time curves of CU. Data represent the mean $\pm S D(n=5)$. (C) Pharmacokinetic parameters of N-PD/CU and PD/CU $(n=5)$.
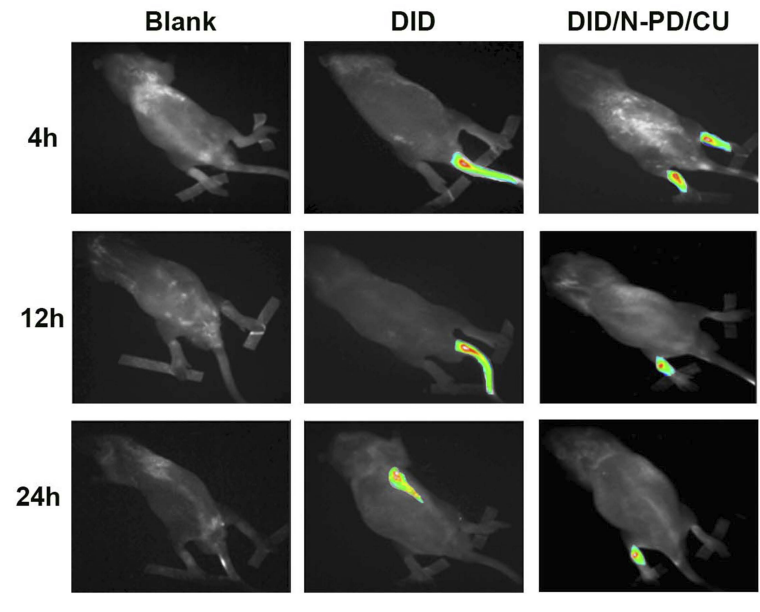

Figure 8 The real-time fluorescence imaging of AIA rats. Rats were administrated by intravenous injection with saline, free DID or DID/N-PD/CU, respectively $(n=3)$.

Abbreviation: DID, Dioctadecyl tetramethyl Indocarbocyanine.
These results suggest greater therapeutic efficacy with N-PD/CU than with single-loaded nanoparticles, either free drug on its own, or a simple mixture of the two drugs.

\section{Discussion}

HSA is an attractive drug carrier, offering high loading capacity for a wide variety of compounds, especially water-insoluble drugs. ${ }^{30}$ We found that the encapsulation and drug loading efficiency of HSA-based nanoparticles were similar regardless of whether they carried PD and CU separately or together. Both drugs were released slowly and sustainably from N-PD/CU, which aids in maintaining therapeutic levels of the drugs in circulation.

Liposomes are perhaps the most extensively studied carrier for targeted delivery of drugs to inflamed joints in 
A

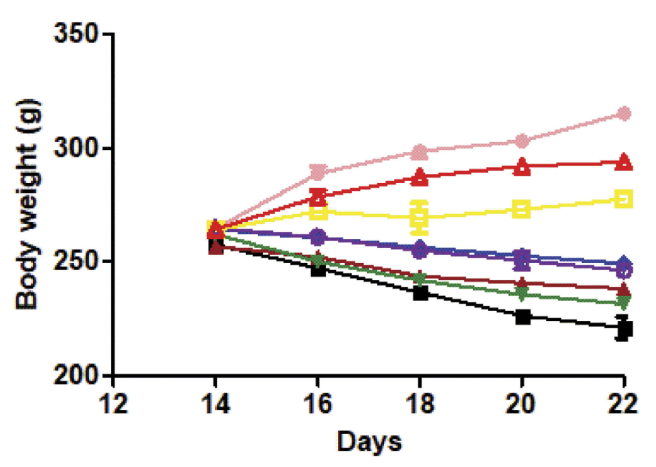

B

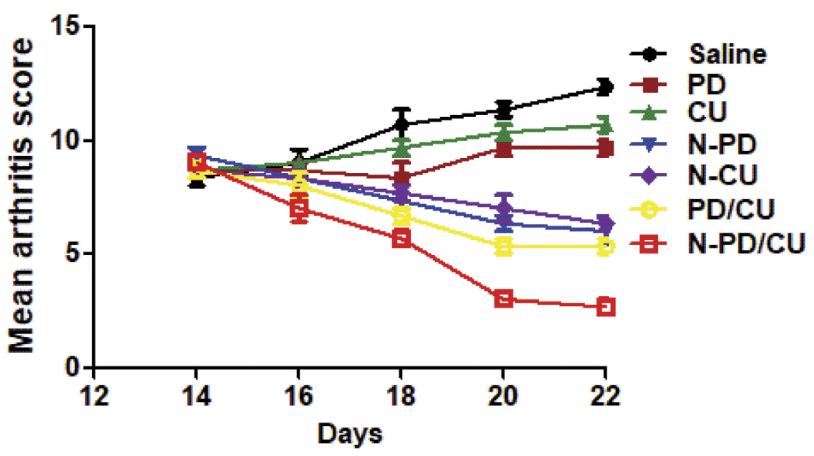

CU

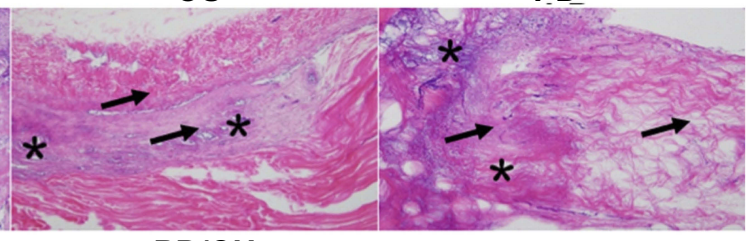

$\mathrm{PD} / \mathrm{CU}$
N-PD/CU

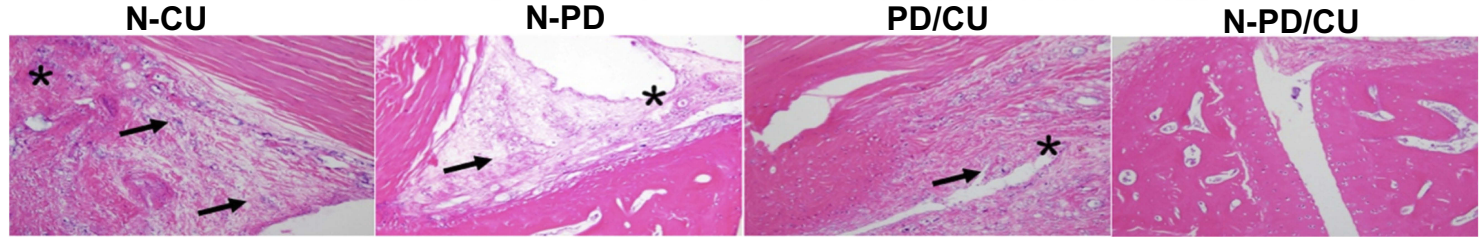

Figure 9 Therapeutic efficacy of nanoparticles in vivo. (A) Body weight variation after given different treatments. (B) Mean arthritis scores were calculated for the joints in the right hind limb following different treatments. Data shown are mean \pm SD $(n=3)$. (C) Photomicrographs of histological sections of ankle joints from animals after different treatments. Arrows indicate finger-like pannus formation; asterisks, bone destruction. Bar, $100 \mathrm{~nm}$.

RA treatment. However, hydrophobic drugs can be encapsulated only into the bilayer of liposomes rather than into their hydrophilic cores. This translates to rapid release in vivo because the lipid bilayer is destabilized. ${ }^{11,31} \mathrm{In}$ our study, we prepared HSA-based nanoparticles by highpressure homogenization, which utilizes high-shear cavitation to form a new disulfide bond in the free sulfhydryl group of albumin, which allows cross-linking into nanoparticles. $^{32}$ In this approach, the drugs formed a tight complex with HSA, with the drugs mainly in the core, E80 and cholesterol helped disperse and stabilize the drug-albumin complexes. These HSA-based nanoparticles avoided the rapid drug release of typical liposomes. In other approachs, ${ }^{33,34}$ researchers have used hyaluronic acid to load hydrophobic drugs such as $\mathrm{CU}$, and phospholipids was used to enhance the stability of the drug-loading system too, obtaining higher drug loading and better stability in vivo than liposomes.

In RA, the formation of synovial hyperplasia vasospasm is associated with early cartilage and bone erosion, and the interface between this vasospasm and cartilage contains a large number of activated macrophages, also known as M1 macrophages. Such macrophages drive the progression of RA by continuously secreting a variety of pro-inflammatory cytokines such as TNF- $\alpha$, IL- $1 \beta$, and IL-6. This makes macrophages a potential therapeutic target in inflammatory diseases. ${ }^{35,36}$ In our work, we used LPS-activated macrophages as an inflammatory model to explore the pharmacodynamic efficacy of N-PD/CU and anti-inflammatory mechanism of PD and $\mathrm{CU}$ separately. N-PD/CU inhibited pro-inflammatory cytokines and promoted production of anti-inflammatory cytokine IL-10 more effectively than other preparations. PD inhibited the production of pro-inflammatory cytokines TNF- $\alpha$ and IL- $1 \beta$ to a greater extent than CU, while CU effectively promoted the production of anti-inflammatory cytokine IL-10. These results suggest that $\mathrm{CU}$ can synergize with PD for combination therapy of RA by simultaneously inhibiting the level of pro-inflammatory cytokines and increasing the level of anti-inflammatory cytokines.

PD and CU reduced the level of IL- 6 to similar extents, which is consistent with some reports that $\mathrm{CU}$ can strongly 

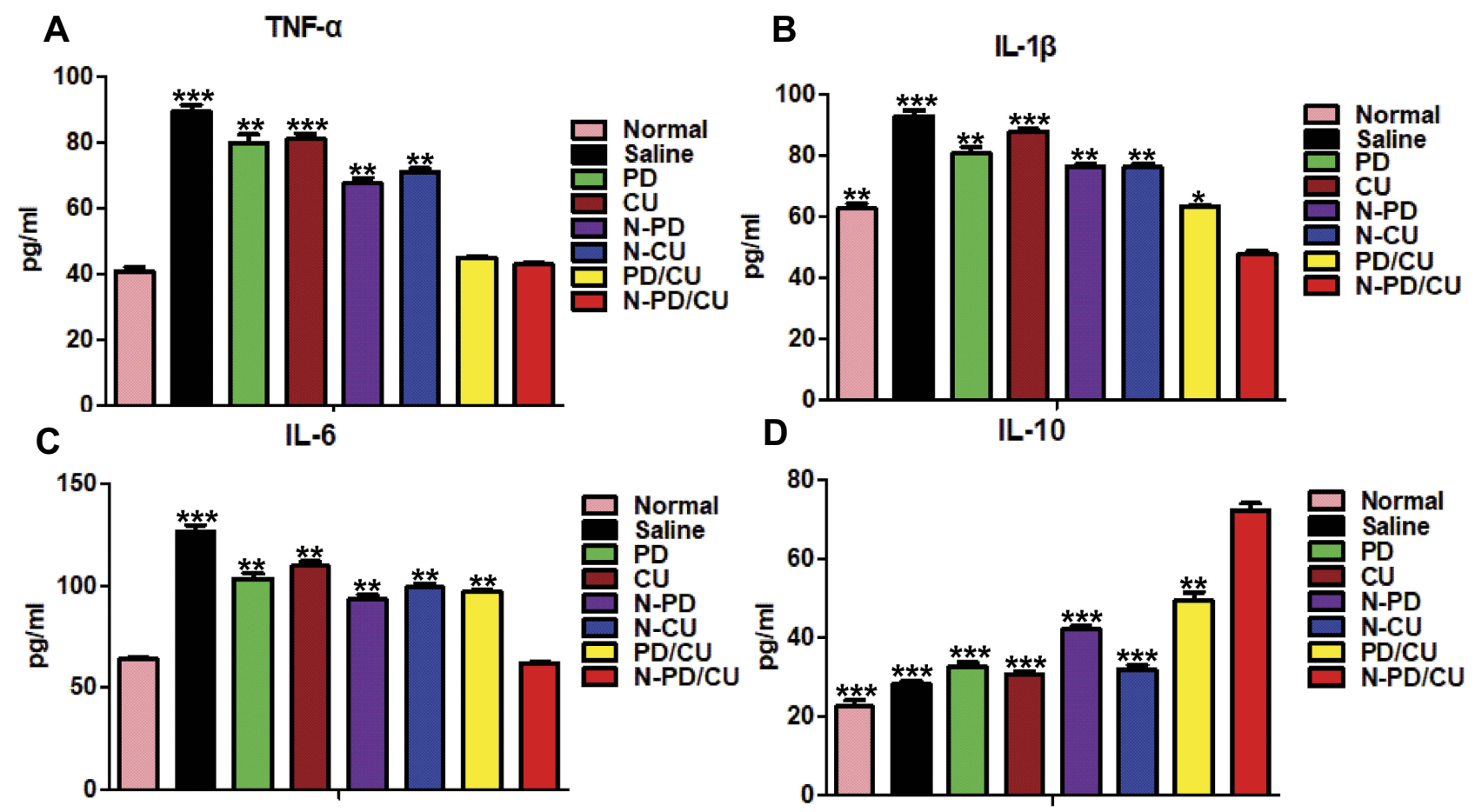

Figure 10 Production of pro-inflammatory cytokines and anti-inflammatory cytokines in arthritic rats treated with different preparations. Healthy, untreated animals served as a control (Normal). Serum levels of (A) TNF- $\alpha,(\mathbf{B}) \mathrm{IL}-\mathrm{I} \boldsymbol{\beta},(\mathbf{C}) \mathrm{IL}-6$ and (D) IL-10 were assayed. Data shown are mean \pm SD ( $\mathrm{n}=3$ ). $* p<0.05, * * p<0.00 \mathrm{I}, * * * p<0.000 \mathrm{I}$ v animals treated with $\mathrm{N}-\mathrm{PD} / \mathrm{CU}$.

reduce IL-6 levels in inflammation. ${ }^{33}$ In addition, Consistent with anti-inflammatory effects, CU reduced oxidative damage in the synovial fluid and improved the production of IL-10, it can also down-regulate pro-inflammatory cytokines such as IL-1, IL-8, as well as monocyte chemotactic protein 1-1 (MCP-1), inducible nitric oxide synthase and nitric oxide. ${ }^{37-39} \mathrm{CU}$ can also modify the activity of $\mathrm{T}$ and B cells, macrophages, neutrophils, dendritic cells and natural killer cells. It inhibits the proliferation of lymphocytes and their secretion of IL-4, IL-5, and granulocyte-macrophage colony-stimulating factor. ${ }^{40}$

$\mathrm{N}-\mathrm{PD} / \mathrm{CU}$ accumulated in joints to a much greater extent than free $\mathrm{PD} / \mathrm{CU}$, suggesting that encapsulating PD and CU with HSA can improve their bioavailability. The joint-targeting ability of $\mathrm{N}-\mathrm{PD} / \mathrm{CU}$ is due to the ELVIS effect. ${ }^{26}$ Therefore, the therapeutic effficacy of PD will be increased, and the side effects caused by systemic distribution will be reduced.

Our results provide further evidence that encapsulating GCs into biomimetic carriers can improve their therapeutic efficacy in AIA rats. These positive results are also due to the stability, high accumulation and sustained drug release of N-PD/CU. Co-delivering GCs with CU reduces the dose of GCs needed, using non-toxic and biocompatible HSA as a nanocarrier reduces the toxicity of drugs to cells in vitro compare to free drugs. Therefore the side effects of GCs reduce, which may improve the clinical application of GCs. Future studies should verify the reduction in side effects in vivo as well as the optimal ratio of PD and CU for combination therapy.

\section{Conclusion}

We successfully prepared HSA-based nanoparticles for codelivery of PD and CU by high-pressure homogenization. The $\mathrm{N}-\mathrm{PD} / \mathrm{CU}$ exhibited synergistic anti-inflammation effect against macrophages and accumulated to a greater extent at inflamed joint sites than single-loaded nanoparticles or a simple mixture of the drugs. This resulted in superior therapeutic effect in rats with adjuvant-induced arthritis. HSAbased nanoparticles show promise for combination chemotherapy.

\section{Abbreviations}

OA, oleic acid; lipoid E80, purified yolk lecithin; RA, rheumatoid arthritis; PD, prednisolone; CU, curcumin; HSA, human serum albumin; N-PD/CU, Co-delivery of prednisolone and curcumin in human serum albumin nanoparticles; N-PD, prednisolone in HSA-based 
nanoparticles; N-CU, curcumin in HAS-based nanoparticles; EE, encapsulation efficiency; DLE, drug loading efficiency; blank, blank HSA-based nanoparticles; MTT, 3-(4,5-dimethylthiazol-2-yl)-2,5-diphenyltetrazolium bromide; DID, Dioctadecyl tetramethyl Indodicarbocyanine; ELISA, Enzyme-linked immunosorbent assay; ELVIS, extravasation through leaky vasculature and subsequent inflammatory cell-mediated sequestration; EPR, enhanced permeability and retention; PBS, phosphate-buffered saline.

\section{Acknowledgements}

This work was supported by Sichuan Science and Technology Program (2017RZ0048) and the National Natural Science Foundation of China (Grant No. 81803478).

\section{Disclosure}

The authors report no conflicts of interest in this work.

\section{References}

1. Lu W, Zhou Z, Naqvi SMAS, Li X. Meta-analysis on efficacy and safety of modified guizhi shaoyao zhimu tang with the western medicines in treating rheumatoid arthritis. Yangtze Med. 2018;02:161-170. doi:10.4236/ym.2018.23017

2. Wang Q, Jiang H, Li Y, et al. Targeting NF-kB signaling with polymeric hybrid micelles that co-deliver siRNA and dexamethasone for arthritis therapy. Biomaterials. 2017;122:10-22. doi:10.1016/j. biomaterials.2017.01.008

3. Crielaard BJ, Rijcken CJF, Quan L, et al. Glucocorticoid-loaded core-cross-linked polymeric micelles with tailorable release kinetics for targeted therapy of rheumatoid arthritis. Angew Chem Int Ed. 2012;51:7254-7258. doi:10.1002/anie.201202713

4. Feldmann M, Maini SRN. Role of cytokines in rheumatoid arthritis an education in pathophysiology and therapeutics. Immunol Rev. 2008;223:7-19. doi:10.1111/j.1600-065X.2008.00626.x

5. Gerli R, Pitzalis C, Lunardi C. The role of $\mathrm{T}$ cell cytokines in modulating joint inflammation in rheumatoid arthritis. IMAJ. 2002;4:949-952.

6. Jain S, Tran TH, Amiji M. Macrophage repolarization with targeted alginate nanoparticles containing IL-10 plasmid DNA for the treatment of experimental arthritis. Biomaterials. 2015;61:162-177. doi:10.1016/j.biomaterials.2015.05.028

7. Li C, Li H, Wang Q, et al. pH-sensitive polymeric micelles for targeted delivery to inflamed joints. $J$ Control Release. 2017;246:133-141. doi:10.1016/j.jconrel.2016.12.027

8. Wang Q, Jiang J, Chen W, et al. Targeted delivery of low-dose dexamethasone using PCL-PEG micelles for effective treatment of rheumatoid arthritis. J Control Release. 2016;230:64-72. doi:10.10 16/j.jconrel.2016.03.035

9. Zhou M, Hou J, Zhong Z, et al. Targeted delivery of hyaluronic acid-coated solid lipid nanoparticles for rheumatoid arthritis therapy. Drug Deliv. 2018;25:716-722. doi:10.1080/10717544.2018.1447050

10. Wang Q, Sun X. Recent advances in nanomedicines for the treatment of rheumatoid arthritis. Biomater Sci. 2017;5:1407-1420. doi:10.10 39/C7BM00254H

11. Quan L, Zhang Y, Crielaard BJ, et al. Nanomedicines for inflammatory arthritis: head-tohead comparison of glucocorticoid-containing polymers, micelles, and liposomes. ACS Nano. 2013;8:458-466. doi: $10.1021 / \mathrm{nn} 4048205$
12. Chuan YP, Zeng BY, O’Sullivan B, et al. Co-delivery of antigen and a lipophilic anti-inflammatory drug to cells via a tailorable nanocarrier emulsion. J Colloid Interface Sci. 2012;368:616-624. doi:10. 1016/j.jcis.2011.11.014

13. Shakibaei M, Schulze-Tanzil G, John T, et al. Curcumin protects human chondrocytes from IL- $1 \beta$-induced inhibition of collagen type II and $\beta 1$-integrin expression and activation of caspase-3: an immunomorphological study. Ann Anat. 2005;187:487-497. doi:10.1016/j. aanat.2005.06.007

14. Mollazadeh $\mathrm{H}$, Cicero AFG, Blesso CN, et al. Immune modulation by curcumin: the role of interleukin-10. Crit Rev Food Sci Nutr. 2019;59:89-101. doi:10.1080/10408398.2017.1358139

15. Ahn JK, Kim S, Hwang J, et al. Metabolomic elucidation of the effects of curcumin on fibroblast-like synoviocytes in rheumatoid arthritis. PLoS One. 2015;10:1-15. doi:10.1371/journal.pone.0145539

16. Arora R, Kuhad A, Kaur IP, Chopra K. Curcumin loaded solid lipid nanoparticles ameliorate adjuvant-induced arthritis in rats. Eur J Pain. 2015;19:940-952. doi:10.1002/ejp.620

17. Banji D, Pinnapureddy J, Banji OJ, et al. Synergistic activity of curcumin with methotrexate in ameliorating Freund's Complete Adjuvant induced arthritis with reduced hepatotoxicity in experimental animals. Eur J Pharmacol. 2011;668:293-298. doi:10.1016/j. ejphar.2011.06.006

18. Kratz F. A clinical update of using albumin as a drug vehicle - a commentary. J Control Release. 2014;190:331-336. doi:10.1016/j. jconrel.2014.03.013

19. Yi X, Lian X, Dong J, et al. Co-delivery of pirarubicin and paclitaxel by human serum albumin nanoparticles to enhance antitumor effect and reduce systemic toxicity in breast cancers. Mol Pharm. 2015;12:4085-4098. doi:10.1021/acs.molpharmaceut.5b00536

20. Elzoghby AO, Samy WM, Elgindy NA. Albumin-based nanoparticles as potential controlled release drug delivery systems. J Control Release. 2012;157:168-182. doi:10.1016/j.jconrel.2011.07.031

21. Elsadek B, Kratz F. Impact of albumin on drug delivery - new applications on the horizon. J Control Release. 2012;157:4-28. doi:10.1016/j.jconrel.2011.09.069

22. Prasad LK, O’Mary H, Cui Z. Nanomedicine delivers promising treatments for rheumatoid arthritis. Nanomedicine. 2015;10: 2063-2074. doi:10.2217/nnm.15.45

23. Wang D, Miller SC, Liu X-M, et al. Novel dexamethasone-HPMA copolymer conjugate and its potential application in treatment of rheumatoid arthritis. Arthritis Res Ther. 2007;9(1):R2. doi:10.1186/ar2106

24. Wang Q, Li Y, Chen X, et al. Optimized in vivo performance of acid-liable micelles for the treatment of rheumatoid arthritis by one single injection. Nano Res. 2018;12:421-428. doi:10.1007/s12274-018-2233-3

25. Wang Y, Liu Z, Li T, et al. Enhanced therapeutic effect of RGD-modified polymeric micelles loaded with low-dose methotrexate and nimesulide on rheumatoid arthritis. Theranostics. 2019;9:708-720. doi:10.7150/thno.30418

26. Quan LD, Purdue PE, Liu XM, et al. Development of a macromolecular prodrug for the treatment of inflammatory arthritis: mechanisms involved in arthrotropism and sustained therapeutic efficacy. Arthritis Res Ther. 2010;12:R170. doi:10.1186/ar3130

27. Fasano M, Curry S, Terreno E, et al. The extraordinary ligand binding properties of human serum albumin. IUBMB Life. 2005;57:787-796. doi:10.1080/15216540500404093

28. Tao-Tao T, Lin-Li L, Wang B, et al. Employing macrophage-derived microvesicle for kidney-targeted delivery of dexamethasone: an efficient therapeutic strategy agaist renal inflammation and fibrosis. Theranostics. 2019;16:4740-4755.

29. Yang M, Feng X, Ding J, Chang F, Chen X. Nanotherapeutics relieve rheumatoid arthritis. $J$ Control Release. 2017;252:108-124. doi:10.1016/j.jconrel.2017.02.032

30. Yuan F, Quan LD, Cui L, et al. Development of macromolecular prodrug for rheumatoid arthritis. Adv Drug Deliv Rev. 2012;64:1205-1219. doi:10.1016/j.addr.2012.03.006 
31. Metselaar JM, Den Berg WB, Holthuysen AE, et al. Liposomal targeting of glucocorticoids to synovial lining cells strongly increases therapeutic benefit in collagen type II arthritis. Ann Rheum Dis. 2004;63(4):348-353. doi:10.1136/ard.2003.009944

32. He W, Lv Y, Zhao Y, et al. Core-shell structured gel-nanocarriers for sustained drug release and enhanced antitumor effect. Int J Pharm. 2015;484(1-2):163-171. doi:10.1016/j.ijpharm.2015.02.053

33. Manca ML, Lattuada D, Valenti D, et al. Potential therapeutic effect of curcumin loaded hyalurosomes against inflammatory and oxidative processes involved in the pathogenesis of rheumatoid arthritis: the use of fibroblast-like synovial cells cultured in synovial fluid. Eur J Pharm Biopharm. 2019;136:84-92. doi:10.1016/j.ejpb.2019.01.012

34. Manca ML, Castangia I, Zaru M, et al. Development of curcumin loaded sodium hyaluronate immobilized vesicles (hyalurosomes) and their potential on skin inflammation and wound restoring. Biomaterials. 2015;71:100-109. doi:10.1016/j.biomaterials.2015.08.034

35. Kinne W, Brauer R, Stuhlmuller B, Palombo-Kinne E, Burmeste G. Macrophages in rheumatoid arthritis. Arthritis Res. 2000;2 2189-2202. doi:10.1186/ar86
36. Lawrence T, Natoli G. Transcriptional regulation of macrophage polarization: enabling diversity with identity. Nat Rev Immunol. 2011;11:750-761. doi:10.1038/nri3088

37. Sundar Dhilip Kumar S, Houreld NN, Abrahamse H. Therapeutic potential and recent advances of curcumin in the treatment of aging-associated diseases. Molecules. 2018;23(4):835-849. doi:10.33 90/molecules23040835

38. Zheng Z, Sun Y, Liu Z, et al. The effect of curcumin and its nanoformulation on adjuvant-induced arthritis in rats. Drug Des Devel Ther. 2015;9:4931-4942. doi:10.2147/DDDT.S90147

39. Khalifé S, Zafarullah M. Molecular targets of natural health products in arthritis. Arthritis Res Ther. 2011;13:102-105. doi:10.1186/ar3222

40. Zhao H, Lu H, Gong T, et al. Nanoemulsion loaded with lycobetaine-oleic acid ionic complex: physicochemical characteristics, in vitro, in vivo evaluation, and antitumor activity. Int $J$ Nanomed. 2013;8:1959-1973.
International Journal of Nanomedicine

\section{Publish your work in this journal}

The International Journal of Nanomedicine is an international, peerreviewed journal focusing on the application of nanotechnology in diagnostics, therapeutics, and drug delivery systems throughout the biomedical field. This journal is indexed on PubMed Central, MedLine, CAS, SciSearch ${ }^{\circledR}$, Current Contents ${ }^{\circledR} /$ Clinical Medicine,
Dovepress

Journal Citation Reports/Science Edition, EMBase, Scopus and the Elsevier Bibliographic databases. The manuscript management system is completely online and includes a very quick and fair peer-review system, which is all easy to use. Visit http://www.dovepress.com/ testimonials.php to read real quotes from published authors. 